\title{
Good food versus bad food: the role of sterols and polyunsaturated fatty acids in determining growth and reproduction of Daphnia magna
}

\author{
Dominik Martin-Creuzburg • Eric von Elert
}

\begin{abstract}
The absence of dietary sterols and polyunsaturated fatty acids (PUFAs) has been shown to affect the performance of the freshwater herbivore Daphnia. Here, we compared somatic growth rates and clutch sizes of Daphnia magna reared on a diet of low food quality (Synechococcus elongatus) and of high food quality (Cryptomonas sp.) and investigated if and to what extent the absence of sterols or PUFAs in the cyanobacterium S. elongatus accounts for the observed differences in food quality. The supplementation of $S$. elongatus with cell-free lipid extracts (fatty acids, sterols, total lipids) obtained from the flagellate Cryptomonas sp. suggested that the superior food quality of Cryptomonas sp. is predominantly, but not completely, a combined effect of its sterol and PUFA composition. Our laboratory study suggests that somatic growth of $D$. magna feeding on S. elongatus is primarily constrained by the absence of sterols, whereas egg production is primarily limited by the absence of long chain PUFAs.
\end{abstract}

Keywords Sterols - Polyunsaturated fatty acids . Liposomes $\cdot$ Growth $\cdot$ Reproduction

\section{Martin Creuzburg (ه)}

Limnological Institute, University of Konstanz,

Mainaustrasse 252, 78464 Constance, Germany

e mail: Dominik.Martin Creuzburg@uni konstanz.de

E. von Elert

Zoological Institute, University of Cologne,

Weyertal 119, 50923 Cologne, Germany

\section{Introduction}

Cladocerans of the genus Daphnia are a key component of freshwater food webs. Due to their high grazing activity on the phytoplankton they are significantly involved in transferring mass and energy (expressed as carbon) from primary producers to higher trophic levels (e.g., Leibold 1989; Carpenter et al. 2001). However, the carbon transfer efficiency across the phytoplankton Daphnia interface is often constrained by the predominance of nutritionally inadequate phytoplankton. Nutritional inadequacy can be attributed to morphological properties that hamper ingestion or digestion (e.g., filaments or colony formation, digestion-resistant cell walls), toxicity, and/or to a deficiency in elemental (mostly phosphorus) or biochemical nutrients (Sterner and Elser 2002; Wilson et al. 2006; Martin-Creuzburg et al. 2008).

In the recent years, food-quality research has increasingly focused on the biochemical nutrient requirements of the major zooplankton taxa. In particular, a dietary deficiency in essential lipids, i.e., polyunsaturated fatty acids (PUFAs) and sterols, has been shown to affect the performance of crustacean grazers (Müller-Navarra et al. 2000; Von Elert 2002; Ravet et al. 2003; Martin-Creuzburg et al. 2005a). Crustaceans, like all arthropods, are incapable of synthesizing long chain PUFAs and sterols de novo, and therefore require a dietary source of these lipids to satisfy their physiological demands (Harrison 1990; Grieneisen 1994). PUFAs and sterols are both 
integral parts of cell membranes and both serve as precursors of many bioactive molecules. The long chain PUFAs, arachidonic acid (ARA; 20:4n-6) and eicosapentaenoic acid (EPA; 20:5n-3), for instance, are precursors of prostaglandins which, among other things, may have several functions in arthropod reproduction (Harrison 1990; Stanley 2006). Sterols, on the other hand, are precursors of steroid hormones, such as ecdysteroids, which are involved in the process of molting (Grieneisen 1994; Martin-Creuzburg et al. 2007).

In pevious studies, we supplemented the steroland PUFA-free picocyanobacterium Synechococcus elongatus with single purified sterols and demonstrated that the well-known nutritional inadequacy of this picocyanobacterium is primarily due to the absence of sterols, i.e., somatic growth of daphnids was significantly improved by sterol supplementation (Martin-Creuzburg and Von Elert 2004; MartinCreuzburg et al. 2005a, b). The absence of PUFAs in $S$. elongatus was found to be of minor importance for somatic growth but may potentially affect egg production in Daphnia, as indicated by increased clutch sizes when a $S$. elongatus diet was supplemented with cholesterol and EPA simultaneously (Martin-Creuzburg et al. 2008).

During the present study, we conducted standardized growth experiments with Daphnia magna feeding on $S$. elongatus and the flagellate Cryptomonas sp., which is ranked as superior food for Daphnia (Weers and Gulati 1997; Martin-Creuzburg et al. 2005b, 2008). In contrast to the previous studies described above, we supplemented $S$. elongatus with lipid enriched liposomes prepared from different lipid extracts derived from Cryptomonas sp. (fatty acid extract, sterol extract, total lipid extract), to investigate the effect of naturally occurring mixtures of sterols and fatty acids on growth and reproduction of D. magna.

\section{Materials and methods}

\section{Cultivation of the food organisms}

The green alga Scenedesmus obliquus (SAG 276-3a) was used as food for the stock cultures of the daphnids. It was grown in batch cultures in Cyano medium (Jüttner et al. 1983) and harvested in the late- exponential growth phase. For the growth experiments, Synechococcus elongatus (SAG 89.79) was cultured semi-continuously in Cyano medium $\left(20^{\circ} \mathrm{C}\right.$; illumination at $80 \mu \mathrm{mol}$ quanta $\mathrm{m}^{-2} \mathrm{~s}^{-1}$ ) at a dilution rate of $0.25 \mathrm{day}^{-1}$ in aerated 5-1 vessels. Cryptomonas sp. (SAG 26.80) was grown in modified WC medium with vitamins (Guillard 1975) at $20^{\circ} \mathrm{C}$ with an illumination at $120 \mu \mathrm{mol}$ quanta $\mathrm{m}^{-2} \mathrm{~s}^{-1}$. Carbon concentrations of the autotrophic food suspensions were estimated from photometric light extinctions $(800 \mathrm{~nm})$ and from previously established regressions between carbon and light extinction determined in dilution series of each food suspension.

Preparation of lipid extracts of Cryptomonas sp. (total lipids, fatty acids, sterols)

Three pre-combusted GF/F filters (Whatman, 47-mm diameter, particle retention $0.7 \mu \mathrm{m}$ ) were each loaded with $20 \mathrm{mg}$ particulate organic carbon (POC), i.e., three filters with $20 \mathrm{mg}$ POC, one for each extract. Lipids were extracted from the filters three times using a mixture of dichloromethane/methanol $(2: 1, v / v)$. The pooled cell-free extracts were evaporated with nitrogen to dryness and resuspended in $4 \mathrm{ml}$ ethanol (three total lipid extracts). The sterol extract was prepared from one total lipid extract by saponification with $0.2 \mathrm{~mol}^{-1}$ methanolic $\mathrm{KOH}\left(70^{\circ} \mathrm{C}, 1 \mathrm{~h}\right)$ and partitioning of the sterols into iso-hexan:diethyl ether $(9: 1, v / v)$. The fatty acid extract was prepared from another total lipid extract after saponification and partitioning of neutral lipids into iso-hexan:diethyl ether as described above and by acidification (HCL, $30 \%, \mathrm{pH}<2$ ) of the residual $\mathrm{KOH}$ fraction and extraction of the free fatty acids with diethyl ether. Subsequently, the lipid extracts (total lipids, fatty acids, sterols) were evaporated to dryness using a rotary evaporator and dissolved in $4 \mathrm{ml}$ of chloroform.

Liposome preparation

Substantially, liposome stock suspensions were prepared by the method described by Martin-Creuzburg et al. (2008). Instead of single purified lipids, however, the lipid extracts of Cryptomonas sp. were used for preparation. Briefly, $3 \mathrm{mg}$ of 1-palmitoyl-2-oleoylphosphatidylglycerol (POPG) and $7 \mathrm{mg}$ of 1-palmitoyl2-oleoyl-phosphatidylcholin (POPC, Lipoid, Germany) were dissolved in an aliquot of chloroform. Sterol or 
PUFA containing liposomes were prepared by adding the total lipid extract, the sterol extract or the fatty acid extract of Cryptomonas sp., respectively. The resulting suspensions were dried using a rotary evaporator, dissolved in $10 \mathrm{ml}$ buffer $\left(20 \mathrm{mmol} \mathrm{I}^{-1} \mathrm{NaP}_{\mathrm{i}}\right.$, $\left.150 \mathrm{mmol} \mathrm{l}^{-1} \mathrm{NaCl}, \mathrm{pH} 7\right)$ and incubated in a rotary shaker (100 revolutions $\mathrm{min}^{-1}$ ) for $30 \mathrm{~min}$. Subsequently, the liposome suspensions were sonicated in an ultrasonic bath, and excess free lipids were removed by washing the liposomes in fresh buffer using an ultraspeed centrifuge $\left(150,000 \times \mathrm{g}, 90 \mathrm{~min}, 4^{\circ} \mathrm{C}\right)$. The liposome stock suspensions were stored at $-20^{\circ} \mathrm{C}$. Prior to the addition of liposomes to the experimental beakers, the liposome stock suspensions were again sonicated ( $2 \mathrm{~min}$ ). Finally, $300 \mu \mathrm{l}$ of a liposome stock suspension $\left(\sim 1 \times 10^{6}\right.$ liposomes $\mathrm{ml}^{-1}$, mean diameter $\sim 4.8 \mu \mathrm{m}$ ) was used as a food supplement in each beaker.

\section{Analyses}

For the analysis of fatty acids and sterols, ca. $0.5 \mathrm{mg}$ particulate organic carbon (POC) of the food suspensions was filtered separately onto precombusted GF/F filters (Whatman, $25 \mathrm{~mm}$ ). The total lipids were extracted three times from filters with dichloromethane/methanol $(2: 1, \mathrm{v} / \mathrm{v})$, and the pooled cell-free extracts were evaporated to dryness with nitrogen. The fatty acid and sterol content of the liposomes was analyzed directly in $100 \mu \mathrm{l}$ of the liposome stock suspensions. The lipid extracts were transesterified with $3 \mathrm{~mol} \mathrm{l}^{-1}$ methanolic $\mathrm{HCL}\left(60^{\circ} \mathrm{C}, 15 \mathrm{~min}\right)$ for the analysis of fatty acids or saponified with $0.2 \mathrm{~mol} \mathrm{l}^{-1}$ methanolic $\mathrm{KOH}\left(70^{\circ} \mathrm{C}, 1 \mathrm{~h}\right)$ for the analysis of sterols. Subsequently, fatty acid methyl esters (FAMEs) were extracted three times with $2 \mathrm{ml}$ of iso-hexan; the neutral lipids were partitioned into iso-hexan:diethyl ether $(9: 1, \mathrm{v} / \mathrm{v})$. The lipid containing fraction was evaporated to dryness under nitrogen and resuspended in $1020 \mu \mathrm{l}$ iso-hexan. Lipids were analyzed by gas chromatography on a HP 6890 GC equipped with a flame ionization detector and a DB225 (J\&W Scientific) capillary column to analyze FAMEs or with a HP-5 (Agilent) capillary column to analyze sterols. Details of GC configurations are given elsewhere (Von Elert 2002 for fatty acids; MartinCreuzburg and Von Elert 2004 for sterols). Lipids were quantified by comparing with internal standards (C17:0 ME and C23:0 ME; $5 \alpha$-Cholestan) and identified by their retention times and their mass spectra, which were recorded with a gas chromatograph-mass spectrometer (Finnigan MAT GCQ) equipped with a fused-silica capillary column (DB225MS, J\&W for FAMEs; DB-5MS, Agilent for sterols). Sterols were analyzed in their free form and as their trimethylsilyl derivatives. Spectra were recorded between 50 and 600 atomic mass units $(\mathrm{amu})$ in the EI ionization mode. The detection limit was $20 \mathrm{ng}$ of fatty acid or sterol. The absolute amount of each lipid was related to the POC, which was determined using a NCS-2500 analyzer (ThermoQuest $\mathrm{GmbH}$, Egelsbach, Germany).

\section{Daphnia growth experiment}

Growth experiments were conducted with third-clutch juveniles (born within $8 \mathrm{~h}$ ) of a clone of Daphnia magna (originally isolated from Großer Binnensee, Lampert 1991). Stock cultures of Daphnia were raised in filtered lake water obtained from Lake Constance $(0.2 \mu \mathrm{m}$ pore-sized membrane filter) and saturating concentrations of $S$. obliquus. The growth experiments were carried out at $20^{\circ} \mathrm{C}$ in glass beakers filled with $200 \mathrm{ml}$ of filtered $(<0.2 \mu \mathrm{m})$ lake water supplemented with $2 \mathrm{mg} \mathrm{C}^{-1}$ of algal or cyanobacterial carbon and the prepared liposomes. The treatments were as follows: S. elongatus, S. elongatus supplemented with either control liposomes or liposomes prepared with the different lipid extracts (fatty acid, sterols, total lipids), and Cryptomonas sp. Each treatment consisted of three beakers (replicates) with six D. magna per beaker. Animals were transferred daily into new beakers with freshly prepared food suspensions. The experimental animals were subsampled at the beginning and at the end of an experiment, dried for $24 \mathrm{~h}$, and weighed on an electronic balance (Mettler UMT 2; $\pm 0.1 \mu \mathrm{g}$ ). Growth rates $(g)$ were determined as the increase in dry mass from day 0 $\left(M_{0}\right)$ to day $6\left(M_{6}\right)$ of the experimental period using the equation: $g=\left(\ln M_{6}-\ln M_{0}\right) t^{-1}$. Eggs were counted in the brood chamber of each female at the end of an experiment using a dissecting microscope.

Data analysis

The somatic growth rates and clutch sizes of Daphnia magna were analyzed by one-way analyses of variance 
(ANOVA). ANOVAs were carried out using the General Linear Model module of STATISTICA 6.0 (StatSoft Inc., Tulsa, Okla., USA). Raw data met the assumption of homogeneity of variance; treatment effects were tested by Tukey's HSD post hoc tests.

\section{Results}

Lipid analyses

The lipid composition of Synechococcus elongatus was characterized by high amounts of short-chain saturated and monounsaturated fatty acid, and by the absence of PUFAs and sterols (Table 1). In contrast, Cryptomonas sp. contained high amounts of $n-3$ PUFAs, such as $18: 3 n-3,18: 4 n-3$, and 20:5n-3. Stigmasterol (24-ethylcholesta-5,22-dien-3 $\beta$-ol) and epibrassicasterol (24-methylcholesta-5,22-dien-3 $\beta$-ol) were the principal sterols found in Cryptomonas sp. (Table 1). Epibrassicasterol, the $24 \alpha$-epimer of brassicasterol, has been previously found in cryptophycean

Table 1 Fatty acid and sterol content of Synechococcus elongatus and Cryptomonas sp.

\begin{tabular}{|c|c|c|}
\hline & $\begin{array}{l}\text { Synechococcus } \\
\text { elongatus }\left(\mu \mathrm{g} \mathrm{m} \mathrm{C}^{-1}\right)\end{array}$ & $\begin{array}{l}\text { Cryptomonas sp. } \\
\left(\mu \mathrm{g} \mathrm{m} \mathrm{C}^{-1}\right)\end{array}$ \\
\hline C16:0 & $22.13 \pm 0.41$ & $20.41 \pm 0.98$ \\
\hline $\mathrm{C} 16: \ln 7$ & $40.89 \pm 0.12$ & $5.48 \pm 0.64$ \\
\hline $\mathrm{C} 17: \ln 7$ & $0.62 \pm 0.08$ & $0.94 \pm 0.04$ \\
\hline $\mathrm{C} 18: 0$ & $4.36 \pm 0.68$ & $4.13 \pm 0.18$ \\
\hline $\mathrm{C} 18: \ln 9$ & $1.44 \pm 0.11$ & $4.32 \pm 0.21$ \\
\hline C18:1n 7 & $2.29 \pm 0.17$ & $8.91 \pm 0.28$ \\
\hline C18:2n 6 & ND & $18.68 \pm 0.22$ \\
\hline C18:3n 6 & ND & $0.89 \pm 0.06$ \\
\hline $\mathrm{C} 18: 3 n \quad 3$ & ND & $74.96 \pm 0.58$ \\
\hline $\mathrm{C} 18: 4 n \quad 3$ & ND & $39.24 \pm 0.51$ \\
\hline $\mathrm{C} 20: 1 n 9$ & ND & $0.52 \pm 0.09$ \\
\hline $\mathrm{C} 20: 4 n \quad 6$ & ND & $0.60 \pm 0.18$ \\
\hline $\mathrm{C} 20: 3 n \quad 3$ & ND & $0.96 \pm 0.01$ \\
\hline $\mathrm{C} 20: 5 n \quad 3$ & ND & $34.48 \pm 0.20$ \\
\hline $\mathrm{C} 22: 6 n \quad 3$ & ND & $4.28 \pm 0.16$ \\
\hline$\left(\begin{array}{ll}n & 3\end{array}\right) /\left(\begin{array}{ll}n & 6\end{array}\right)$ & ND & 7.63 \\
\hline Epibrassicasterol & ND & $2.43 \pm 0.24$ \\
\hline Stigmasterol & ND & $3.81 \pm 0.17$ \\
\hline
\end{tabular}

Data are means of three replicates $\pm \mathrm{SD}$

$N D$ not detectable algae, such as Cryptomonas sp. and Rhodomonas sp. (Goad 1981; Gladu et al. 1990). Although we did not determine the stereochemistry of the side chain at C-24, a $24 \alpha$-configuration was also assumed.

The prepared liposomes contained significant amounts of palmitic acid (C16:0) and oleic acid (C18:1n-9), which are both components of the phospholipids POPG and POPC (Table 2). In addition, liposomes prepared in the presence of the total lipid extract of Cryptomonas sp. contained high amounts of all major fatty acids of this flagellate (in particular 18:3n-3, 18:4n-3, and 20:5n-3) together with the two sterols epibrassicasterol and stigmasterol. Liposomes prepared from the fatty acid or sterol extract of Cryptomonas sp. contained considerably lower amounts of fatty acids but hardly differed in their fatty acid composition. Sterols, however, were absent in liposomes prepared from the fatty acid extract of Cryptomonas sp. (Table 2).

\section{Daphnia growth experiment}

Growth of Daphnia magna on unsupplemented S. elongatus was poor (Fig. 1). Supplementation of $S$. elongatus with control liposomes or liposomes prepared with the fatty acid extract of Cryptomonas sp. did not affect somatic growth rates of $D$. magna. Growth was significantly improved, however, when $S$. elongatus was supplemented with liposomes prepared with the sterol extract of Cryptomonas sp. (Tukey's HSD, $P<0.05$; ANOVA, $F_{5,12}=3,499$, $P<0.001$; Fig. 1). Supplementation of $S$. elongatus with liposomes prepared from the total lipid extract of Cryptomonas sp. did not further improve growth of D. magna, but led to significantly increased clutch sizes compared to supplementation with the sterol extract (Tukey's HSD, $P<0.05$; ANOVA, $F_{2,6}=$ $147, P<0.001)$. D. magna did not produce eggs when grown on unsupplemented $S$. elongatus or $S$. elongatus supplemented with control liposomes or liposomes prepared with a fatty acid extract of the flagellate. Growth rates and clutch sizes of D. magna were highest when grown on Cryptomonas sp. (Fig. 1).

\section{Discussion}

We compared somatic growth rates and clutch sizes of D. magna reared on a diet of low (S. elongatus) 
Table 2 Fatty acid and sterol content of the liposomes prepared from lipid extracts of Cryptomonas sp. (fatty acids, sterols, total lipids)

\begin{tabular}{|c|c|c|c|c|}
\hline & $\begin{array}{l}\text { Liposomes } \\
\text { (control) }[\mu \mathrm{g}]\end{array}$ & $\begin{array}{l}\text { Liposomes + fatty } \\
\text { acids }[\mu \mathrm{g}]\end{array}$ & Liposomes + sterols $[\mu \mathrm{g}]$ & $\begin{array}{l}\text { Liposomes + total } \\
\text { lipids }[\mu \mathrm{g}]\end{array}$ \\
\hline C16:0 & $108.34 \pm 3.64$ & $83.63 \pm 0.47$ & $55.85 \pm 0.60$ & $81.44 \pm 1.43$ \\
\hline $\mathrm{C} 16: 1 n \quad 7$ & ND & $0.95 \pm 0.09$ & $0.73 \pm 0.01$ & $2.30 \pm 0.03$ \\
\hline $\mathrm{C} 17: \ln 7$ & ND & ND & ND & ND \\
\hline C18:0 & ND & $3.10 \pm 0.05$ & $2.44 \pm 0.02$ & $3.13 \pm 0.03$ \\
\hline $\mathrm{C} 18: \ln 9$ & $101.23 \pm 1.88$ & $70.90 \pm 1.78$ & $60.58 \pm 0.51$ & $67.93 \pm 2.33$ \\
\hline C18:1n 7 & ND & $0.65 \pm 0.01$ & $0.71 \pm 0.00$ & $1.64 \pm 0.06$ \\
\hline $\mathrm{C} 18: 2 n \quad 6$ & ND & $2.82 \pm 0.05$ & $2.68 \pm 0.00$ & $7.65 \pm 0.17$ \\
\hline C18:3n 6 & ND & ND & ND & ND \\
\hline $\mathrm{C} 18: 3 n \quad 3$ & ND & $15.78 \pm 0.21$ & $14.40 \pm 0.01$ & $50.24 \pm 0.74$ \\
\hline $\mathrm{C} 18: 4 n \quad 3$ & ND & $13.23 \pm 0.13$ & $11.91 \pm 0.01$ & $45.61 \pm 0.86$ \\
\hline $\mathrm{C} 20: 1 n \quad 9$ & ND & ND & ND & ND \\
\hline $\mathrm{C} 20: 4 n \quad 6$ & ND & ND & ND & ND \\
\hline $\mathrm{C} 20: 3 n \quad 3$ & ND & ND & ND & ND \\
\hline $\mathrm{C} 20: 5 n \quad 3$ & ND & $10.36 \pm 0.30$ & $10.01 \pm 0.00$ & $33.48 \pm 0.93$ \\
\hline $\mathrm{C} 22: 6 n \quad 3$ & ND & $1.40 \pm 0.20$ & $1.06 \pm 0.01$ & $2.28 \pm 0.12$ \\
\hline$\left(\begin{array}{ll}n & 3\end{array}\right) /\left(\begin{array}{ll}n & 6\end{array}\right)$ & ND & 14.41 & 13.95 & 17.20 \\
\hline Epibrassicasterol & ND & ND & $2.34 \pm 0.52$ & $3.63 \pm 0.53$ \\
\hline Stigmasterol & ND & ND & $5.04 \pm 1.28$ & $8.05 \pm 1.43$ \\
\hline
\end{tabular}

Data indicate the amount that was supplied daily to D. magna with the different liposome suspensions (300 $\mu 1$ in 200 ml lake water, containing $2 \mathrm{mg} \mathrm{C}^{-1}$ of $S$. elongatus). Data are means of three replicates $\pm \mathrm{SD}$

$N D$ not detectable

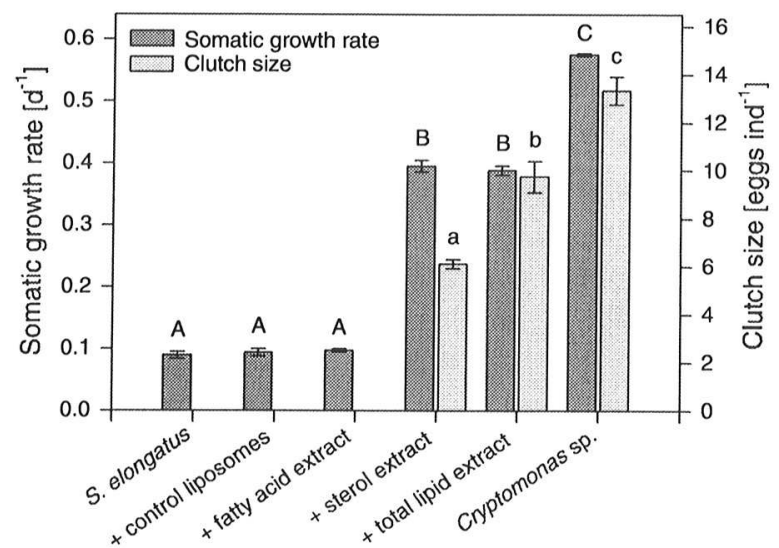

Fig. 1 Juvenile somatic growth rates of Daphnia magna grown on Synechococcus elongatus and on S. elongatus supplemented with liposomes prepared from lipid extracts of Cryptomonas sp. (fatty acids, sterols, total lipids). Growth on Cryptomonas sp. is given for reference. Data are means of three replicates per treatment; error bars indicate SD. Bars labeled with the same letters are not significantly different (Tukey's HSD, $P<0.05$ following ANOVA) and of high (Cryptomonas sp.) food quality. By supplementation of the cyanobacterium $S$. elongatus with cell-free lipid extracts (fatty acids, sterols, total lipids) obtained from the flagellate Cryptomonas sp., we analyzed if and to what extent the absence of certain fatty acids and sterols in S. elongatus accounts for the observed differences in food quality. In previous studies, the supplementation of $S$. elongatus with single PUFAs (Martin-Creuzburg et al. 2008) or with deliberate mixtures of PUFAs (Von Elert and Wolffrom 2001; Von Elert et al. 2003) failed to improve the growth of Daphnia sp., which has been interpreted to indicate that the low quality of $S$. elongatus was not due to the absence of these PUFAs. Here, to exclude that the PUFAs previously supplemented to $S$. elongatus were inadequate to support Daphnia sp., growth, we used a natural mixture of PUFAs from a high quality food organism (Cryptomonas sp.) for supplementation. The supplementation of $S$. elongatus with the fatty acid extract of Cryptomonas sp., containing all major fatty acids 
of the flagellate, including the $n-3$ PUFAs $18: 3 n-3$, $18: 4 n-3,20: 5 n-3$, and $22: 6 n-3$, did not improve somatic growth of $D$. magna, indicating that the absence of PUFAs in $S$. elongatus is not the predominant food quality constraint.

In contrast to the supplementation with the fatty acid extract, the supplementation of $S$. elongatus with the sterol extract of Cryptomonas sp., containing stigmasterol and epibrassicasterol as the major sterols, significantly improved somatic growth of $D$. magna. Thus, our study clearly shows a prevailing sterol limitation of $D$. magna feeding on the cyanobacterium $S$. elongatus. Such a primary sterol limitation of Daphnia sp. on S. elongatus has also been demonstrated during our previous studies (cp. Von Elert et al. 2003; Martin-Creuzburg and Von Elert 2004; Martin-Creuzburg et al. 2005a, 2008) by supplementing single purified sterols, mostly cholesterol. However, cholesterol is the end-product of sterol assimilation in Daphnia sp., and it has not been tested before whether naturally occurring mixtures of sterols from autotrophic phytoplankton can reduce sterol limitation in Daphnia sp., by serving as precursors for the synthesis of cholesterol (cp. Martin-Creuzburg and Von Elert 2004). In this respect, our data suggest that at least one of the two principal sterols present in Cryptomonas sp. (stigmasterol, epibrassicasterol) can be converted into cholesterol, which had released $D$. magna from sterol limitation.

The supplementation of $S$. elongatus with the total lipid extract of Cryptomonas sp., containing PUFAs and sterols, did not increase the growth rate of $D$. magna beyond $0.4 \mathrm{day}^{-1}$. This implies that 0.4 day $^{-1}$ is the maximum growth rate that can be obtained by lipid supplementation of the cyanobacterium $S$. elongatus. Interestingly, a similar somatic growth rate was observed by simultaneously supplementing the cyanobacterium with cholesterol and EPA (Martin-Creuzburg et al. 2008), which suggests that the food quality of the cyanobacterium is constrained only by cholesterol and EPA (and potential precursors) but not by additional lipids. However, the addition of the total lipid extract of Cryptomonas sp., to the $S$. elongatus food suspension led to significantly increased clutch sizes compared with the addition of the sterol extract. The liposomes prepared from the total lipid extract of Cryptomonas sp. contained significantly more PUFAs, in particular
$18: 3 n-3,20: 5 n-3$, and $22: 6 n-3$, than those prepared from the sterol extract, implying that the availability of dietary $n-3$ PUFAs enhances egg production in D. magna. This is in good agreement with the finding that $D$. magna allocates dietary 20:5n-3 (EPA) preferentially into the eggs (Becker and Boersma 2005; Wacker and Martin-Creuzburg 2007), and the implication that EPA plays a crucial role in Daphnia reproduction (Martin-Creuzburg et al. 2008). Notably, however, the threshold levels for saturation with $18: 3 n-3$ and $20: 5 n-3$ are presumably rather low (Müller-Navarra 1995; Wacker and Von Elert 2001; Becker and Boersma 2005; Gladyshev et al. 2008). Hence, the fatty acid requirements of $D$. magna might have been satisfied already by the fatty acids present in the sterol extract, suggesting that the significant increase in clutch sizes obtained by the addition of the total lipid extract is due to other unknown lipids or lipid-like compounds present in Cryptomonas sp. and absent in S. elongatus.

Although the addition of sterols and total lipids (sterols included) improved the food quality of $S$. elongatus for D. magna, growth rates observed on the supplemented diets were much lower than those achieved on a sole diet of Cryptomonas sp. (Fig. 1). Cryptomonas sp. contained considerably smaller amounts of sterols and $n-3$ PUFAs $(18: 3 n-3$, $18: 4 n-3,20: 5 n-3,22: 6 n-3)$ than were provided with the liposomes prepared with the total lipid extract, which suggests that D. magna was sufficiently supplied with sterols and $n-3$ PUFAs. In addition to $n-3$ PUFAs, Cryptomonas sp. contained small amounts of the $n-6$ PUFA 20:4n-6 (ARA), which was absent in the liposome supplemented diets. Becker and Boersma (2005) reported that even small amounts of ARA supplemented to an ARA-free diet significantly improved the growth of $D$. magna. Moreover, ARA is a precursor of prostaglandins, which have been shown to be important in the reproduction of arthropods (Harrison 1990; Stanley 2006). Thus, even the minute amounts of ARA present in Cryptomonas sp. may have contributed to the high food quality of this flagellate. It is also possible that the fitness of daphnids feeding on Cryptomonas sp. is positively affected by the $n-3 / n-6$ ratio of PUFAs. Likewise, the ratio of saturated to unsaturated fatty acids may be important, because saturated fatty acids provided in excess (such as C16:0 in the liposome 
supplemented diets) may have detrimental effects on daphnids (Wacker and Martin-Creuzburg 2007). It should also be noted that liposomes are a considerable source of carbon and phosphorus and that a supplementation with liposomes will provide a grazer with extra energy (Martin-Creuzburg et al. 2008). However, because the supplementation with control liposomes (prepared without lipids from Cryptomonas sp.) and with liposomes prepared from the fatty acid extract of Cryptomonas sp. did not improve somatic growth, a carbon and phosphorus limitation of $D$. magna is unlikely. In general, S. elongatus is very rich in phosphorus and nitrogen and thus already provides Daphnia with high amounts of these elements, certainly with higher amounts than are provided by Cryptomonas sp. (e.g., DeMott et al. 2004; MartinCreuzburg et al. 2008). Hence, in the present study, a limitation of $D$. magna by nitrogen is also improbable (but see Gladyshev et al. 2006).

The fact that the daphnids fed Cryptomonas sp. performed significantly better than those animals fed with $S$. elongatus which was supplemented with the total lipid extract from the flagellate, suggests that other factors, e.g., essential amino acids or vitamins, become limiting once the sterol and PUFA requirements are satiated. Nevertheless, the main conclusions of our study are: (1) the superior food quality of Cryptomonas sp. for D. magna when compared with $S$. elongatus is predominantly a combined effect of its sterol and PUFA composition; and (2) that the absence of sterols in a $S$. elongatus diet primarily constrains somatic growth of $D$. magna, whereas the absence of long chain PUFAs primarily limits egg production. Further laboratory and field studies are required to reveal the significance of these findings under field conditions and to disentangle the complex patterns of nutritional challenges daphnids face in natural environments and their potential consequences for food web processes.

Acknowledgments We thank $P$. Merkel for excellent technical assistance. This work was supported by the German Research Foundation (DFG, EI 179/4 2).

\section{References}

Becker C, Boersma M (2005) Differential effects of phos phorus and fatty acids on Daphnia magna growth and reproduction. Limnol Oceanogr 50:388 397
Carpenter SR, Cole JJ, Hodgson JR, Kitchell JF, Pace ML, Bade D, Cottingham KL, Essington TE, Houser JN, Schindler DE (2001) Trophic cascades, nutrients, and lake produc tivity: whole lake experiments. Ecol Monogr 71: 163186

DeMott WR, Edington JR, Tessier AJ (2004) Testing zoo plankton food limitation across gradients of depth and productivity in small stratified lakes. Limnol Oceanogr 49: 14081416

Gladu PK, Patterson GW, Wikfors GH, Chitwood DJ, Lusby WR (1990) The occurrence of brassicasterol and epibrassicas terol in the chromophycota. Comp Biochem Physiol B 97:491 494. doi:10.1016/0305 0491(90)90149 N

Gladyshev MI, Sushchik NN, Dubovskaya OP, Makhutova ON, Kalachova GS (2006) Influence of sestonic elemental and essential fatty acid contents in a eutrophic reservoir in Siberia on population growth of Daphnia (longispina group). J Plankton Res 28:907 917. doi:10.1093/plankt/fbl028

Gladyshev MI, Sushchik NN, Dubovskaya OP, Makhutova ON, Kalachova GS (2008) Growth rate of Daphnia feeding on seston in a Siberian reservoir: the role of essential fatty acid. Aquat Ecol 42:617 627. doi:10.1007/s10452 00791467

Goad LJ (1981) Sterol biosynthesis and metabolism in marine invertebrates. Pure Appl Chem 51:837 852. doi:10.1351/ pac 198153040837

Grieneisen ML (1994) Recent advances in our knowledge of ecdysteroid biosynthesis in insects and crustaceans. Insect Biochem Mol Biol 24:115 132. doi:10.1016/0965 1748 (94) 900787

Guillard RR (1975) Cultures of phytoplankton for feeding of marine invertebrates. In: Smith WL, Chanley MH (eds) Culture of marine invertebrate animals. Plenum Press, New York, pp 2660

Harrison KE (1990) The role of nutrition in maturation, repro duction and embryonic development of decapod crustaceans: a review. J Shellfish Res 9:1 28

Jüttner F, Leonhardt J, Möhren S (1983) Environmental factors affecting the formation of mesityloxid, dimethylallylic alcohol and other volatile compounds excreted by Anabaena cylindrica. J Gen Microbiol 129:407 412

Lampert W (1991) The dynamics of Daphnia in a shallow lake. Verh Int Verein Limnol 24:795 798

Leibold MA (1989) Resource edibility and the effects of pre dators and productivity on the outcome of trophic interactions. Am Nat 134:922 949. doi:10.1086/285022

Martin Creuzburg D, von Elert E (2004) Impact of 10 dietary sterols on growth and reproduction of Daphnia galeata. J Chem Ecol 30:483 500. doi:10.1023/B:JOEC.00000186 24.94689.95

Martin Creuzburg D, Wacker A, von Elert E (2005a) Life history consequences of sterol availability in the aquatic keystone species Daphnia. Oecologia 144:362 372. doi: 10.1007/s0044200500908

Martin Creuzburg D, Bec A, von Elert E (2005b) Trophic upgrading of picocyanobacterial carbon by ciliates for nutrition of Daphnia magna. Aquat Microb Ecol 41:271 280. doi:10.3354/ame041271

Martin Creuzburg D, Westerlund SA, Hoffmann KH (2007) Ecdysteroid levels in Daphnia magna during a molt cycle: determination by radioimmunoassay (RIA) and liquid chromatography mass spectrometry (LC MS). Gen Comp Endocrinol 151:66 71. doi:10.1016/j.ygcen.2006.11.015 
Martin Creuzburg D, von Elert E, Hoffmann KH (2008) Nutritional constraints at the cyanobacteria Daphnia magna interface: the role of sterols. Limnol Oceanogr 53: 456468

Müller Navarra DC (1995) Evidence that a highly unsaturated fatty acid limits Daphnia growth in nature. Arch Hydro biol 132:297 307

Müller Navarra DC, Brett M, Liston AM, Goldman CR (2000) A highly unsaturated fatty acid predicts carbon transfer between primary producers and consumers. Nature 403: 74 77. doi: $10.1038 / 47469$

Ravet JL, Brett MT, Müller Navarra DC (2003) A test of the role of polyunsaturated fatty acids in phytoplankton food quality for Daphnia using liposome supplementation. Limnol Oceanogr 48:1938 1947

Stanley D (2006) Prostaglandins and other eicosanoids in insects: biological significance. Annu Rev Entomol 51: 25 44. doi:10.1146/annurev.ento.51.110104.151021

Sterner RW, Elser JJ (2002) Ecological stoichiometry: the biology of elements from molecules to the biosphere. Princeton University Press, Princeton

Von Elert E (2002) Determination of limiting polyunsaturated fatty acids in Daphnia galeata using a new method to enrich food algae with single fatty acids. Limnol Ocea nogr 47:1764 1773
Von Elert E, Wolffrom T (2001) Supplementation of cyano bacterial food with polyunsaturated fatty acids does not improve growth of Daphnia. Limnol Oceanogr 46:1552 1558

Von Elert E, Martin Creuzburg D, Le Coz JR (2003) Absence of sterols constrains carbon transfer between cyanobac teria and a freshwater herbivore (Daphnia galeata). Proc R Soc Lond B Biol Sci 270:1209 1214. doi:10.1098/ rspb.2003.2357

Wacker A, Martin Creuzburg D (2007) Allocation of essential lipids in Daphnia magna during exposure to poor food quality. Funct Ecol 21:738 747. doi:10.1111/j.1365 2435 . 2007.01274.x

Wacker A, von Elert E (2001) Polyunsaturated fatty acids: evidence for non substitutable biochemical resources in Daphnia galeata. Ecology 82:2507 2520

Weers PMM, Gulati RD (1997) Effects of the addition of polyunsaturated fatty acids to the diet on the growth and fecundity of Daphnia galeata. Freshw Biol 38:721 729. doi:10.1046/j.1365 2427.1997.00237.x

Wilson AE, Sarnelle O, Tillmanns AR (2006) Effects of cyanobacterial toxicity and morphology on the population growth of freshwater zooplankton: meta analyses of lab oratory experiments. Limnol Oceanogr 51:1915 1924 